\title{
Correction to: Family Functional Formulations as Guides to Psychological Treatment
}

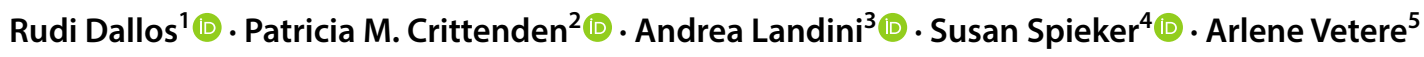

Published online: 30 January 2020

○) Springer Science+Business Media, LLC, part of Springer Nature 2020

\section{Correction to: Contemporary Family Therapy https://doi.org/10.1007/s10591-019-09525-6}

The original version of this article unfortunately contained errors in the "Abstract", "Introduction", "Formulation" and "References."

In the Abstract, the expansion of DMM should be capitalized, i.e. Dynamic-Maturational Model of Attachment and Adaptation.

The first paragraph of the paper is in error and should be replaced with the following text:

Systemic family therapists have long sought to identify how family dynamics are causally related to different types of problems and to consequent implications for improving treatment (Minuchin et al. 1978; Bowen 1971). These efforts diminished because they focused too closely on the direct relation of family patterns to symptoms (Hoffman 1981). Recently, however, there is a resurgence of interest, for example, Kaslow and Patterson (2006) describe attempts to develop a systemic model of relational diagnosis for inclusion in DSM. Using the Dynamic-Maturational Model of Attachment and Adaptation (DMM), we suggest focusing on family members' strategies for self- and child-protection; doing so shifts the priority from connecting patterns with

The original article can be found online at https://doi.org/10.1007/ s10591-019-09525-6.

Patricia M. Crittenden

crittenden@patcrittenden.com

1 Department of Clinical Psychology, University of Plymouth, Plymouth, UK

2 Family Relations Institute, 9481 SW 147 St, Miami, Fl 33176, USA

3 Family Relations Institute, Reggio Emilia, Italy

4 Child, Family, and Population Health Nursing, University of Washington, Seattle, WA, USA

5 VID Specialized University, Oslo, Norway symptoms to describing the function of patterns to protect family members.

Considering danger and protective behavior is important because psychological treatments are less effective than would be expected and, according to some authorities, have shown little or no improvement in outcomes in the recent three decades (Insel 2014; Kaslow and Patterson 2006; Wampold 2015). When family therapy is considered specifically, there is an extensive evidence base, comprised of systematic reviews and meta-analyses of published randomized controlled trials testing effectiveness of family therapy and systemic interventions for child and adult problems (Carr 2014, 2019; Retzlaff et al. 2013; von Sydow et al. 2010). These studies indicate generally positive effects sizes across a variety of family therapy approaches in several different cultures. Depending on the review and particular problem, effect sizes for family therapy compared to treatment as usual were significant but small to moderate, meaning a substantial minority of patients did not improve. These reviews also noted a tendency for effect sizes in later publications to be lower than initial publications, suggesting some weakening of approaches as they moved from developers to the general practitioner. Because these reviews include only published studies, there is a possibility that publication bias, i.e., the strong tendency for researchers to fail to write up and publish null findings (Franco et al. 2014), would further weaken effect sizes for family therapy interventions.

The heading of Sect. 1 contains a typo; the word 'sematic' should read as 'semantic'.

The following text should be added as a last paragraph of Example 1 Physical Abuse under 'Formulating Pete's Problem' subsection:

The problem was obviously in the father, but its basis was unclear. Treatment needed to discover Pete's hidden triggers and the means of disarming them.

Also, the following text should be added as a last paragraph on Example 2: Behavior Problems under 'Formulating the Family's Problem' subsection: 
The problem to be resolved was differentiating the functions of the brothers' use of disruptive behavior and discovering how the parents could respond productively.

The final paragraph of Example 4: Autism under 'Formulating the Family's Problem' subsection is in error.

The incorrect text read: 'Penny's mother showed some indication of reflection in her AAI, but it was only about understanding the influence that her parents' childhood experiences had had on their parenting of her. She was not able to reflect on her own behavior.'

The correct text should read: 'The problem to be resolved was enabling Penny's mother to address two past generations of denied neglect so as to transform the vicious cycle into a virtuous cycle of increasing acceptance by Penny of her mother's protective authority.'

In the reference list, the following citations should be added:

\section{References}

Carr, A. (2014). The evidence base for family therapy and systemic interventions for child-focused problems. Journal of
Family Psychology, 36, 107-157. https://doi.org/10.1111/14676427.12032.

Carr, A. (2019). Family therapy and systemic interventions for childfocused problems: The current evidence base. Journal of Family Therapy, 41, 153-213. https://doi.org/10.1111/1467-6427.12226.

Franco, A., Malhotra, N., \& Simonovits, G. (2014). Social science. Publication bias in the social sciences: Unlocking the file drawer. Science. https://doi.org/10.1126/science.1255484.

Hoffman, L. (1981). Foundations of family therapy: A conceptual framework for systems change. New York: Basic Books.

Retzlaff, R., von Snydow, K., Beher, S., Haun, M. W., \& Schweitzer, J. (2013). The efficacy of systemic therapy for internalizing and other disorders of childhood and adolescence: A systematic review of 38 randomized trials. Family Process. https://doi.org/10.1111/ famp. 12041.

von Sydow, K., Beher, S., Schweitzer, J., \& Retzlaff, R. (2010). The efficacy of systemic therapy with adult patients: A meta-content analysis of 38 randomized controlled trials. Family Process. https ://doi.org/10.1111/j.1545-5300.2010.01334.x.

Publisher's Note Springer Nature remains neutral with regard to jurisdictional claims in published maps and institutional affiliations. 